\title{
Un modelo pedagógico de educación universitaria a distancia para estudiantes en prisión: perspectivas y desafíos
}

\section{A pedagogical model for students deprived of freedom in a distance higher education university or institution: perspectives and challenges}

\author{
William Murillo Leiva ${ }^{1}$ \\ Universidad Estatal a Distancia, Costa Rica
}

Resumen. El planteamiento de un modelo pedagógico de educación universitaria a distancia para estudiantes en prisión implica la consideración de múltiples y variados aspectos para posibilitar la construcción de un modelo acorde con las necesidades y especificidades de la población y el contexto penitenciario. Este modelo se enfoca en el contexto penitenciario costarricense, el cual guarda relaciones y semejanzas, generalmente, con la mayoría de los contextos penitenciarios de los países latinoamericanos. Además, reflexionando acerca de la posibilidad de construir un modelo pedagógico de educación universitaria a distancia para estudiantes en prisiones de Costa Rica y aquellas con condiciones semejantes, este documento pretende destacar algunos de los principales elementos -a consideración del autor y con base en la referencia bibliográfica- por tomar en cuenta en el diseño y en la problematización de dicho modelo.

Palabras clave. Modelo pedagógico, educación, prisión, privación de libertad.

\begin{abstract}
The proposal of a pedagogical model of distance university education for students in prison implies the consideration of multiple and diverse components to enable the construction of a model according to the needs and specificities of the population and the prison context; emphasizing in the Costa Rican penitentiary context, which shows relationships and similarities -generally- with most prison contexts in Latin American countries. In addition, reflecting on the possibility of creating an educational model of distance university education for students in prison in Costa Rica and other places with similar conditions, this paper intends to highlight some of the main elements - according to the author's views and the bibliographical references - to be considered in the design and the problematization of said model.
\end{abstract}

Keywords. Pedagogical model, education, prison, deprivation of freedom.

${ }^{1}$ William Murillo Leiva. Investigador en el Programa de Investigación en Fundamentos de la Educación a Distancia (PROIFED), Universidad Estatal a Distancia de Costa Rica. Dirección electrónica: willmurilloleiva@gmail.com 


\section{Introducción}

La construcción de un modelo pedagógico de educación universitaria a distancia para personas en prisión involucra una multiplicidad de retos por enfrentar, aspectos por considerar y tareas por realizar para lograr una propuesta correcta, integral y acorde con las especificidades y necesidades de la población en cuestión.

Cuando se hace referencia a las históricamente denominadas "prisiones", "centros penitenciarios", "penales" o "cárceles", en Costa Rica se han llamado Centros de Atención Institucional (CAI), o inclusive y de manera más reciente, Unidades de Atención Integral (UAI); las cuales se diferencian de acuerdo con sus modelos de trabajo y de atención. Pese a las diferencias en cuanto su denominación técnica y de modelos de trabajo, en términos generales, se hace referencia a las instituciones del sistema penitenciario en Costa Rica, establecidas para recluir a las personas en conflicto con la ley y condenadas al internamiento en dichos espacios por la comisión de algún hecho tipificado como delito, y así, comprobado y determinado por la instancia judicial.

A estas personas, dependiendo de lainstitución destacada donde se encuentren, se les podría denominar "privados o privadas de libertad", "internos e internas o residentes", entre otras y cuando así corresponda. Sin embargo, comparten la imposibilidad de abandonar la institución hasta que cumplan con su condena o cuando, de acuerdo con el criterio técnico, profesional y judicial que corresponda, puedan disfrutar de algún beneficio estipulado por ley que les permita la salida de las instituciones señaladas.

En relación con lo anterior, se plantean en este documento algunos de los aspectos que, con base en la investigación bibliográfica y su respectivo análisis, deben tomarse en consideración al pensar, al aproximarse y al diseñar un modelo pedagógico de educación a distancia para estudiantes universitarios recluidos en estas instituciones del sistema penitenciario.

Así, el objetivo de esta investigación es explorar elementos trascendentales y necesarios cuando se plantea un modelo pedagógico de educación a distancia para estudiantes universitarios en prisión. Y, a partir de lo anterior, reflexionar acerca de la importancia de estos elementos y de la posibilidad de implementarlos y de promoverlos. Además, se busca conocer aspectos transversales y oportunos para el estudio universitario en el contexto penitenciario y se enfatiza en la modalidad de estudios a distancia.

En cuanto a la metodología, corresponde a una investigación de antecedentes y aspectos teóricos relacionados. La investigación es teórica a partir de la revisión bibliográfica proveniente de diversas bases de datos, de artículos científicos, así como de trabajos finales de graduación y libros de texto. De acuerdo con Barahona (2012), “[...] podemos concebir la investigación teórica como la actividad sistemática de elaborar, construir, reconstruir, explorar y analizar críticamente los cuerpos conceptuales (esto es, teóricos) en que se enmarcan las distintas áreas del saber". (p. 8). Además, es de carácter exploratorio, pues analiza y reflexiona sobre una cuestión poco estudiada y posicionada en el quehacer académico y científico.

Sobresalen como resultados la necesidad de incorporar internet $\mathrm{y}$, especialmente, las nuevas tecnologías de la información en los procesos educativos de estos contextos. El acceso a la información, mediante nuevas tecnologías, es trascendental a la hora de repensar los procesos educativos en el sistema penitenciario, ya que potencia el disfrute y el desarrollo de las actividades académicas propias de la actividad. También, destaca la imperante necesidad de establecer procesos educativos acordes y coherentes con las necesidades y especificidades del contexto y de la población. 
Resalta, relacionado con lo anterior, la importancia de pensar e implementar un diseño de modelo pedagógico que entienda y fundamente su estructura, procedimientos y funcionamiento de acuerdo con la comprensión de la educación como derecho humano. Y como un derecho humano complejo, se desarrolla en la medida en que una diversidad de condiciones se garanticen y se ejecuten.

En este mismo sentido, se determina que es trascendental aproximarse a la construcción de un modelo pedagógico que promueva una educación que potencie el desarrollo de un ser humano capaz de problematizar y de ser crítico ante su entorno y su propio ser. En sí, lo anterior debería ser uno de los fines trasversales del quehacer educativo en cualquier contexto, no solo el penitenciario.

Los resultados se obtienen a partir de información de diversas procedencias y de multiplicidad de países y pretenden reflexionar, de manera general, al respecto de la educación universitaria y universitaria a distancia en el contexto de los centros penales. Sin embargo, hay una mirada, -de análisis, de reflexión y de recomendaciones- especializada y enfocada en la posibilidad de un modelo pedagógico de educación universitaria a distancia especializado para estudiantes del contexto destacado y de la Universidad Estatal a Distancia (UNED) de Costa Rica.

De acuerdo con lo anterior, algunas de las principales recomendaciones que, a partir de este trabajo, se generan responden a la necesidad de iniciar -en primera instancia- espacios de investigación, de reflexión y de mejora continua acerca de los procesos educativos establecidos en las instituciones mencionadas; y específicamente, a la posibilidad de estructurar un modelo pedagógico pensado para la comunidad estudiantil de dichas instituciones.
Por último, se considera muy valiosa la recomendación de establecer, diseñar e implementar un modelo de educación universitaria a distancia para personas en prisión que sea coherente y acorde con las especificidades del contexto.

\section{Desarrollo}

En primer lugar, ¿por qué pensar y plantear un modelo de educación universitaria a distancia para personas en prisión? La respuesta es que es una cuestión de derechos humanos.

Toda persona, de acuerdo con la Declaración Universal de Derechos Humanos, en su artículo número 26, tiene derecho a la educación; así dice la declaración:

Toda persona tiene derecho a la educación. La educación debe ser gratuita, al menos en lo concerniente a la instrucción elemental y fundamental. La instrucción elemental será obligatoria. La instrucción técnica y profesional habrá de ser generalizada; el acceso a los estudios superiores será igual para todos, en función de los méritos respectivos (Organización de las Naciones Unidas, 2015, p. 54).

Y, además, señala:

La educación tendrá por objeto el pleno desarrollo de la personalidad humana y el fortalecimiento del respeto a los derechos humanos $\mathrm{y}$ a las libertades fundamentales; favorecerá la comprensión, la tolerancia y la amistad entre todas las naciones y todos los grupos étnicos o religiosos, y promoverá el desarrollo de las actividades de las Naciones Unidas para el mantenimiento de la paz (ONU, 2015, p. 54).

De acuerdo con lo anterior, la justificación, el fundamento y el pilar central de un modelo pedagógico deeducación universitaria a distancia para personas en prisión ha de ser siempre una perspectiva de derechos humanos. Una perspectiva que reivindique, visualice y promueva el disfrute y la defensa de dicho derecho. En este sentido, Blazich, de Milén y Viedma (2007), destacan que 
La posibilidad de estudiar en estos contextos, va más allá de una preparación del/la interno a la sociedad o un vínculo laboral futuro; tiene que ver con la recuperación de un derecho, el de la educación, y con la normalización de la vida cotidiana durante el cumplimiento de la condena; en este sentido su reconstrucción está relacionada con la satisfacción personal o las expectativas futuras (p.3).

También, señala Tscharf (2009), citado en Moreira, Reis y Machado (2017), que

Una educación para todos, a lo largo de toda la vida y accesible por igual según la naturaleza concreta de todos y cada uno deberá cubrir necesariamente la educación y la formación de los adultos y, por lo tanto, la educación y la formación dentro de las prisiones (p. 48).

Este tipo de educación debe imponerse a visiones, perspectivas y posicionamientos presentes en la sociedad, e inclusive -quizás con alguna frecuencia- en el contexto académico y que limitan la oportunidad y la posibilidad de acceso a la educación por parte de esta población y a partir de la cosa juzgada y por la autoridad competente. Dichas perspectivas actúan casi como una segunda instancia judicial o de administración de la pena, aspecto que, evidentemente, no corresponde.

En este sentido, Manchado (2012), citado en Elvira-Valdez y Durán-Aponte (2014), enfatiza que "La educación en los centros penitenciarios arrastra el estigma de ser tomado en cuenta más como un beneficio para reducir la pena impuesta, que como un derecho humano capaz de reeducar y reinsertar al condenado" (pp. 65-66). Es este punto, quizás el más importante y el que más desafíos plantea a la educación universitaria y a la educación en general, en el contexto penitenciario. La visión y la perspectiva de la educación universitaria en cárcel como derecho son clave para repensar estos procesos educativos por y para la mejora y la innovación. En esta mirada, reside la oportunidad de cambios y de especialización. La tarea no atañe solo a los que- haceres educativos, fundamentos psicopedagógicos o herramientas didácticas, antes de esto, hay que darle la oportunidad a la educación en cárcel de establecerse y de desarrollarse; así, se puede avanzar hacia las consideraciones necesarias y adecuadas para su óptimo desarrollo.

Aquí, vale destacar que el derecho a la educación debe garantizarse en el contexto de una serie de condiciones esenciales. De acuerdo con INEE (2014), se destaca la disponibilidad, la accesibilidad, la adaptabilidad y la aceptabilidad como "mínimo irreductible del derecho a la educación" (INEE, 2014, p. 11).

Las dos primeras A - disponibilidad y accesibilidad - corresponden al derecho a la educación, mientras que las segundas - adaptabilidad y aceptabilidad - refieren al derecho en la educación (Bracho, 2011). Esto significa que la población tiene derecho no sólo a acceder a la escuela sino a que esta ofrezca las condiciones materiales, socioafectivas y pedagógicas necesarias para que efectivamente pueda aprender (INEE, 2014, p. 11).

De acuerdo con los autores, en lo respectivo a la disponibilidad, se hace referencia a la existencia de los espacios o centros educativos y que, entre otras cosas, "[...] cuenten con maestros suficientemente formados; operando en forma regular en una organización; dotadas de infraestructura, mobiliario y equipo indispensables para el logro de los propósitos educativos" (INEE, 2014, p. 11).

En cuanto a la accesibilidad, está claro que la oferta educativa no es suficiente, estableciéndose "[...] que no debe haber barreras de ningún tipo para acceder a la educación" (INEE, 2014, p. 11). Aquí se toma en consideración que las barreras podrían ser de cualquier tipo: económicas, de infraestructura, de exclusión. Además, la adaptabilidad

[...] hace referencia al significado, pertinencia y relevancia de la educación que se ofrece. Los 
contenidos y las formas de enseñanza deben adaptarse a las características de los alumnos en el contexto cultural en el que se trabaja y, de la misma manera, deben ser sensibles a las características individuales de los alumnos, que en todo grupo son diversas (INEE, 2014, p. 12).

Finalmente, "[...] la aceptabilidad representa otro acercamiento a la dimensión de calidad de la educación desde la perspectiva de los estudiantes" (INEE, 2014, p.12). Esto hace referencia a considerar a al estudiantado participante como personas que deben estar satisfechas y a gusto con lo que aprenden en su proceso educativo y constituirse en evaluadores centrales del proceso educativo.

Así, el contexto penitenciario y la educación no escapan a las consideraciones citadas; más bien, deben estar presentes a la hora de planificar los procesos educativos en este contexto, por ende, tienen que permanecer en todo modelo de educación en dichos espacios. Se visualiza, entonces, un modelo pedagógico que se fundamenta en un derecho esencial y además complejo, un derecho que debe desarrollarse en un contexto con una serie de variables que añadan calidad y coherencia al proceso educativo. Esta especialización y búsqueda del cumplimiento de un derecho de manera integral (y no solo por decir que se cumple) es posible, de la mano de la voluntad política y académica que dé lugar a una verdadera educación. En términos generales, la educación es considerada un derecho clave para el logro del desarrollo personal, humano y social. "El derecho a la educación - que no es sino el derecho a aprender - está en el centro de la vida de la persona y de la sociedad. Es lo que permite "el desarrollo armónico de las facultades del ser humano"'" CPEUM (2014), citado en INEE (2014, p. 10.

\section{Un escenario complejo y específico}

Aunado a una esencial reivindicación de derechos humanos y directamente en relación con lo que se mencionó acerca de las consideraciones esenciales para garantizar el derecho a la educación, es importante visualizar y destacar que la experiencia de prisión plantea significativas dificultades y especificidades en torno a la cotidianidad, así como al desarrollo personal y educativo.

[...] la educación en los espacios de la prisión, tendría que constituirse en el arte de crear espacios de libertad en los que redundaran tendencias como yo puedo, yo aprendo, yo sé. Pero, ¿es posible crear, transformar y promover nuevas realidades en estos contextos? (Elvira-Valdez y Durán-Aponte, 2014, p. 65).

En este sentido, la educación y el proceso educativo, así como un modelo pedagógico específico de educación universitaria a distancia para personas en prisión debe ser estructurado de acuerdo con una mirada que atienda a la complejidad y a la especificidad que involucra establecer y desarrollar procesos pedagógicos y académicos en centros penitenciarios. De esta manera, la educación puede establecerse según las necesidades e intereses de la población, con el objetivo de promover el desarrollo de un ser humano integral, crítico y responsable. Relacionado con esto, Blazich, de Milén y Viedma (2007) mencionan que "La educación en centros penitenciarios conforma un escenario altamente complejo, generado por múltiples tensiones que se derivan, entre otras cuestiones, de la articulación entre dos sistemas con marcos normativos diferentes: el sistema educativo y el sistema penitenciario" (p. 3).

De acuerdo con ese escenario, el modelo pedagógico por plantear debe ser bien estructurado, pero al mismo tiempo flexible y, sobre todo, coherente con el contexto penitenciario, su marco normativo y especificidades cotidianas. En esta misma línea, di Franco y Domínguez (2005) problematizan la cuestión de la educación en el contexto penitenciario y enfatizan que el análisis por realizar para establecer mejoras y cambios 
debe ser minucioso y crítico; en consecuencia, se deben evaluar limitaciones que se establecen como obstáculos para, como ellos mencionan, el "conocimiento y la acción" (di Franco y Domínguez, 2005, p. 160). Así lo destacan estos autores:

Reglas institucionales que impiden una línea de acción, calendario escolar particular, tiempos de encuentros de clases, asiduidad, posibilidades físicas de hacer tareas (tener lugar, luz, espacio), elección de los contenidos, historias escolares previas, todas dimensiones sobre las que habría que identificar si actuaban como limitaciones objetivas o subjetivas o no eran obstáculos sobre el conocimiento y sobre la acción (p. 160).

Un modelo con perspectiva de género y libre de discriminación

Continuando con otros aspectos esenciales por considerar en este posible modelo pedagógico, se debe hacer énfasis en que una perspectiva de género y de inclusividad debe tomarse en cuenta y debe fundamentar su diseño y la ejecución. La pena de prisión, como se sabe, no está limitada a la población masculina y, por ende, no se puede excluir de la mirada crítica en torno a la educación a las mujeres. En este sentido, las mujeres en prisión son actoras clave y deben ser activas en su proceso educativo; el modelo, entonces, debe ser responsable y coherente con las especificidades de cada uno y de cada una.

También, y evidentemente, dentro de los centros penitenciarios, existen poblaciones de distintas preferencias y elecciones en cuanto a su sexualidad y género, por ende, tampoco pueden ser invisibilizadas. La población LGBTI tiene que disfrutar de, y tener al alcance, un modelo pedagógico que no discrimine, que incluya y que potencie las habilidades y las capacidades de cada persona en un ambiente libre de coerción, discriminación e irrespeto. Se destaca aquí porque es cotidiana la vulnerabilización de de- rechos a dicha población y esto es incompatible con el modelo pedagógico aquí propuesto, y en todo sentido.

Así, es trascendental que la educación no promueva, reproduzca ni mantenga estereotipos de género y de preferencia sexual, desde una posición de poder que priorice el machismo, el patriarcado o la heteronormatividad.

El debate actual se centra en si la educación para las mujeres y los varones seguirá reproduciendo los estereotipos de género, si continuará y reforzará la socialización temprana recibida en el hogar, o bien, si transmitirá nuevos saberes tecnológicos, pero también contenidos éticos tales como la solidaridad, la tolerancia y el respeto mutuo, componentes necesarios para que todos los ciudadanos, hombres y mujeres, junto con la capacidad para asumir riesgos, tomen decisiones y participen activamente, con autonomía y juicio crítico, en cualquier asunto público (Miranda, 2007, p. 4).

Llama la atención que, aunque el debate destacado en la cita se ha planteado por este autor hace ya diez años, no está acabado en las instituciones carcelarias, quizá, más bien, apenas empieza en este contexto. La educación en contextos penitenciarios de mujeres sigue manteniendo, en algunos casos, una perspectiva de reproducción de roles y de estereotipos denigrantes en la medida en que promueve aprendizajes y labores acordes con la lógica machista en las mujeres prisionalizadas.

Un modelo pedagógico de educación universitaria a distancia para población en prisión que tenga perspectiva de género y fomente la inclusión debe superar este tipo de reproducciones estigmatizadoras. Aquí destaca, por ejemplo, la necesidad de potenciar una oferta académica de educación superior con contenidos que posibiliten el acceso para las mujeres a carreras y especializaciones social e históricamente asignadas a la población masculina. Como mencionan Elvira-Valdez y Durán-Aponte (2014), “[...] en muchos centros penitenciarios se continúa impartiendo 
cursos tradicionales de educación no formal ligados a la condición tradicional con la que ha sido vista la mujer, como el tejido o la cocina" (p. 66).

\section{Educación inclusiva de necesidades especiales}

Parte de la estructuración de un modelo pedagógico de educación universitaria a distancia para población en prisión involucra la existencia y la claridad en torno a la promoción de una educación inclusiva para todas las personas que engloba a quienes tienen algún tipo de discapacidad o condición especial. Álvarez et ál. (2012, citado en Fernández-Morales y Duarte 2016, p. 96), señala que

El movimiento inclusivo considera que los centros educativos deben satisfacer las necesidades de todos los alumnos, sean cuales sean sus características personales, psicológicas o sociales. La inclusión implica transformar la cultura, la organización y las prácticas de los centros para atender a la diversidad de necesidades educativas de todo el alumnado. Es una enseñanza adaptada al estudiante, cuyas acciones van dirigidas a eliminar o minimizar las barreras físicas, personales o institucionales que limitan las oportunidades de aprendizaje, el acceso y la participación en las actividades formativas (p. 96).

Destaca en la cita anterior, que una educación inclusiva minimiza las barreras institucionales que limitan las oportunidades de aprendizaje y que, en el contexto penitenciario y en los distintos centros de detención, se establecen y se manifiestan, de manera clara y constante, en la vida académica de la comunidad estudiantil.

Como se ha mencionado, la institución penitenciaria tiene un marco normativo rígido y estructurado que conlleva múltiples dificultades para la población universitaria, y que es difícil de modificar; sin embargo, la mirada y la propuesta de un modelo pedagógico de educación universitaria a distancia para esa población debe ser crítica, propositiva y con condiciones idóneas, a fin de lograr una educación accesible, idónea, eficaz, de calidad, justa, equitativa y adecuada.

\section{El estudiantado como centro del proceso educativo}

Indistintamente del contexto en el que se encuentra la población estudiantil recluída, su condición jurídica o penitenciaria, en su condición de estudiante y de ser humano cada uno debe ser el centro de sus procesos de educativos. Anderson y Dron (2011), citados en Saez, Domínguez y Mendoza (2014), destacan que

Desde cualquier modelo o planteamiento, lo esencial es que el alumno sea el protagonista en su proceso de aprendizaje. Los sitios Web, libros, materiales de tutoriales, vídeos, de los cuales un estudiante puede aprender, todos funcionan más o menos eficazmente de acuerdo el modo eficaz que estos permiten al alumno a adquirir conocimientos (p. 198).

Tal y como lo indica UNED (2004):

Un modelo centrado en el estudiante debe permitirle, a éste, la libertad de aprovechar al máximo los recursos que se le ofrecen, de planificar el progreso de su aprendizaje y de regular, él mismo, el ritmo y la calidad de sus avances. Esto implica que todos los elementos del modelo pedagógico se piensen para ponerlos a disposición de los estudiantes, de manera que ellos puedan gestionar su propio proceso de formación (p.13).

Si bien es cierto que la condición de persona recluida en un centro penitenciario genera, en muchas ocasiones, miradas estigmatizadoras y alienantes, se enfatiza en que, en el proceso educativo, dichas miradas no deben tener lugar. En este espacio, indiferentemente de quién sea la persona estudiante, una vez que adquiere esta condición debe ocupar un lugar central y prioritario; quizás, incluso, un sitio que no ha ocupado con regularidad tanto dentro como fuera del centro penitenciario. Esto es educativo y transformador por sí mismo. Se recalca lo ya mencionado, la educación en cárcel no es un "beneficio" 
que posibilita ofrecer "algo", lo que sea y como sea que se acerque a educación y que, por ende, obligue o limite al estudiantado a sentirse satisfecho y agradecido con esto.

\section{¿Cuál estudiante?}

El modelo educativo propuesto en este documento debe buscar y facilitar el desarrollo y la potencialización de estudiantes, como hemos mencionado, problematizadores y sensibles. Con habilidades de criticidad y de análisis, “¿Qué se gana con esto? Todo. Se les está enseñando a pensar; es más, se les está recordando que pueden pensar por ellos mismos, que pueden construir su propio conocimiento, que no dependen del opresor para que las cosas sean" (Valverde, 2011, p. 126).

La intención debe ser la de promover la consolidación de un estudiantado capaz de transformar su realidad en busca del mayor crecimiento personal posible. Esta transformación debería resignificar su manera de vincularse consigo mismo y con las demás personas; en general, con su entorno.

Cuando el estudiante se propone luchar contra el avasallamiento, contra la dominación y la visión alienante en que los otros tratan de colocarlo, comienza una lucha perenne por la emancipación de su persona como individuo y como ser capaz. Este estudiante sabrá que puede trascender el modelo y aprender más que cualquiera, asumiendo una actitud crítica, inquisidora, activa, pensante, reflexiva, cuestionadora de todo lo que recibe del docente. Ese estudiante se hará ver como sagaz, inteligente y, sobre todo, libre (Valverde, 2011, p. 127).

En este sentido, Mello (1987), citado en Cammarosano (2011), destaca que la educación en prisión significa pensar en la capacidad de promover un ser humano participante del mundo en el que vive y con consciencia crítica que favorezca la capacidad de cuestionar y de problematizar el mundo.
La interacción y la participación como estrategia metodológica y por medio de las nuevas tecnologías de información

“[...] difícil imaginar un quehacer educativo estimable en las cárceles si quienes participan de sus procesos no se implican activamente en ellos - como protagonistas y no como simples destinatarios de las iniciativas que se promuevan-, con una visión renovada de quiénes son y de cómo han de orientar sus vidas durante y después de estar en prisión" (Caride y Gradai1le, 2013, p. 43).

En la construcción y búsqueda de estudiantes que problematicen y sean el centro del proceso educativo, la participación en construir el aprendizaje es fundamental. En el contexto penitenciario, es igual de significativa. Como se indica en la cita anterior, el estudiantado debe ser protagonista activo de su proceso de aprendizaje, debe buscar el crecimiento y el desarrollo personal. Menciona Coll (1999), citado en Diaz y Hernández (2002):

El alumno es el responsable último de su propio proceso de aprendizaje. Él es quien construye (o más bien reconstruye) los saberes de su grupo cultural, y este puede ser un sujeto activo cuando manipula, explora, descubre o inventa, incluso cuando lee o escucha la exposición de otros (p. 30).

Además, facilitar la interacción y la participación estudiante-estudiante, estudiante-docente y estudiante-contenido, deber ser prioridad en el proceso educativo en condiciones de encierro y, por ende, la responsabilidad es compartida por todos los actores y las actoras implicadas. Esta interacción y participación no solo irrumpe con la cotidianidad y monotonía de la institución carcelaria, sino que también promueve la construcción de aprendizajes significativos.

Aunque la educación a distancia presenta retos, en lo relativo a la interacción y a la participación del estudiantado y, que estos se magnifican 
en una institución penitenciaria, no quiere decir que deba invisibilizarse su relevancia. Por lo tanto, es básico apuntar a los centros penitenciarios y su imperativa necesidad de establecerse como lugar de oportunidades y que busque facilitar procesos educativos participativos. De acuerdo con su discurso manifiesto -y falaz-, esta es, en sí, la función de la cárcel: promover una transformación que potencie a un ser humano.

En ese sentido, los presupuestos metodológicos de un programa de educación para tal contexto, fundamentados en la participación activa del grupo estudiantil y en los vínculos de confianza y de diálogo para la construcción de saberes, podrían carecer de sentido práctico en tanto se procure ajustar al estudiantado a la dinámica penitenciaria. Entonces, es indispensable que este espacio sea humanizado y se efectúen transformaciones que conviertan a la cárcel en una institución educativa (Cammarosano, 2011).

Así pues, las herramientas didácticas de este posible modelo que aquí se problematiza deben establecerse de acuerdo con este objetivo (promover espacios participativos de interacción) y, por lo tanto, promover el diseño y el desarrollo de insumos que movilicen la construcción de aprendizajes compartidos, la interacción y el planteamiento de ideas propias, consultas y cuestionamientos críticos y analíticos hacia los contenidos de aprendizaje.

Aquí el e-learning y la utilización de nuevas tecnologías de información es la clave fundamental

La importancia del acceso al conocimiento necesario -mediante el internet y la utilización de nuevas tecnologías de información- suma uno de los aspectos más significativos en torno a esta posibilidad de construir un modelo pedagógico de educación universitaria especializado para personas en prisión.

El acceso a internet por parte de estudiantes en centros penales es nulo. Sin embargo, la uti- lidad y la eficacia de las herramientas tecnológicas es innegable y la imposibilidad del acceso a internet no elimina, necesariamente, la oportunidad de utilizar herramientas tecnológicas en la educación a distancia en prisiones. Y más allá, la imposibilidad actual del acceso a internet, en algunos contextos penitenciarios, no debería determinar un imposible e inquebrantable acceso en el futuro y con determinadas condiciones.

Entendemos por e-learning, "La aplicación del e-learning supone administrar la educación utilizando la tecnología, videoconferencia interactiva y materiales digitales en la nube, es decir, accesibles en un entorno virtual de aprendizaje" y se destaca que "Un aprendizaje a través de e-learning comprende todas las formas y opciones relativas a la tecnología educativa, habilitadas para actividades de aprendizaje" (Saez, Domínguez y Mendoza, 2014, p. 196).

En el contexto penitenciario, resulta crucial y urgente proporcionar un acceso seguro a contenidos o a plataformas de aprendizaje, que posibiliten un mayor acercamiento a esta cuarta generación de educación a distancia (Moreira, Reis y Machado, 2017).

Los autores citados anteriormente destacan que, para la población estudiante y universitaria en prisión, "las tecnologías digitales son herramientas esenciales para la adquisición y la consolidación de conocimientos" (p. 46). Así, la comunidad de estudiantes "aceptan la integración de las tecnologías digitales en su formación porque les aporta cosas nuevas dentro de la prisión, y porque puede ayudarles de hecho a mejorar la adquisición de conocimientos" (p. 46). De manera general, Pelizzari et ál. (2002), citado en Moreira, Reis y Machado (2017), "argumentan que las características de las tecnologías utilizadas en la educación a distancia -por ejemplo, los ordenadores- fomentan la interacción y son constructivistas, por lo que mejoran el desarrollo del aprendizaje" (p. 46). 
A partir de esa necesidad de las herramientas tecnológicas, plantea desafíos, en consideración, más políticos y de voluntad que prácticos, como ya hemos destacado, la voluntad en el contexto de la privación de libertad es trascendental. En realidad, debe existir claridad en torno a la necesidad de implementar herramientas didácticas y nuevas tecnologías de información en los procesos educativos del contexto penitenciario. Y, con base en esto, se debe movilizar las acciones para su implementación.

Si se potencia el aprendizaje y la interacción $\mathrm{y}$, por ende, el desarrollo de habilidades y de capacidades; además, si se busca un modelo pedagógico que realmente impacte y mejore las condiciones de estudio en las prisiones, ¿por qué no implementar cambios idóneos y adecuados? Es decir, coherentes con los objetivos planteados y los resultados esperados.

En este sentido, algunas de las dificultades que se destacan a la hora de impulsar el e-learning en las prisiones son los siguientes: recursos financieros limitados; población meta diversa y con variedad de necesidades; condiciones institucionales que dificultan la implementación, por ejemplo, restricciones en el ingreso a distintas facilidades dentro del penal; cambios y fluctuaciones en la población; poca flexibilidad del sistema penitenciario que, con argumentos de seguridad, suele detener el avance en innovación y desarrollo; entre otros. (Hammerschick, 2010). Sin embargo, algunas de las múltiples contribuciones que brinda el e-learning a los procesos educativos en prisión son estas:

- Extensión de tópicos y de contenidos

- Involucramiento de más prisioneros en actividades educativas y de capacitación

- $\quad$ Permiso al grupo de estudiantes para llevar a cabo investigación y acceder a materiales y al conocimiento que, de otra manera, no podrían acceder
- Como factor de motivación

- La facilitación del mantenimiento y de la constancia del estudiantado en los programas educativos que ha iniciado y ante posibles traslados dentro del sistema penitenciario, o inclusive, ante la salida de prisión (Hammerschick, 2010).

Al respecto de esto, vale destacar los esfuerzos citados por Moreira, Reis y Machado (2017) acerca del diseño de un "Un campus virtual especialmente diseñado para la población reclusa, con acceso seguro y contenidos específicos que permitan desarrollar actividades educativas y formativas de educación a distancia y e-learning" (pp. 47-48), por parte de la Universidad Abierta y la Dirección General de Servicios Penitenciarios y Reinserción Social de Portugal. Los autores destacan que el campus "contará con diferentes servicios online, y se basará en una plataforma tecnológica que brinda tecnologías de e-learning y e-management que facilitan los procesos educativos, académicos, administrativos y de ciudadanía digital" (Moreira, Reis y Machado, 2017, p. 48). En este mismo sentido, varios países europeos (Alemania, Austria, Bélgica, entre otros) han desarrollado, en mayor o menor medida, acciones tendientes a la implementación de las nuevas tecnologías de educación en los centros penales (Hammerschick, 2010).

Esa implementación de nuevas tecnologías en centros penales consolida la noción de que es posible y necesario desarrollar procesos pedagógicos basados en e-learning y nuevas tecnologías; además, la importancia que adquiere, en un modelo pedagógico de educación a distancia en prisiones, la comunicación y el acceso a la información. En la cárcel, el hermetismo y la restricción es la norma, por ende, el acceso a la información y al conocimiento se ve afectado gracias a las particularidades del encierro; en el e-learning, se encuentra una manera de mitigar dichas limitaciones. 
Está claro que la cuestión de seguridad en el desarrollo de nuevas tecnologías de información y del acceso a internet, dentro de las prisiones, es trascendental y que no atender dicha necesidad puede involucrar, precisamente, a que iniciativas en esta línea terminen en el fracaso, además de poner en riesgo la innovación y el desarrollo posible.

Por lo tanto, la mirada no debe basarse en ignorar las múltiples necesidades y especificidades que se deben tomar en cuenta para la implementación exitosa de estos esfuerzos en prisión, incluso en lo que a seguridad corresponde; más bien, es imperativo incluirlas y enfrentarlas con innovación y pericia para posibilitar un desarrollo óptimo.

Mientras el acceso a internet sea nulo o restringido para las personas internas de los centros penitenciarios, las herramientas y las nuevas tecnologías de la información pueden ser facilitadas por medio del tutor o del encargado universitario en prisión. Así, se facilita el acceso a herramientas como las videoconferencias, los podcast, los videotutoriales, las bases de datos.

La comunidad docente como facilitadora de los procesos pedagógicos

Enel contextodeloqueseha venidomencionando, el grupo docente universitario, en contexto de privación de libertad, tiene un papel significativo como facilitador del aprendizaje, de la interacción y de las herramientas didácticas. Sobre todo, de nuevas oportunidades y perspectivas de vida.

Tal y como destaca Cammarosano (2011), para el profesorado, el trabajo en prisión es enriquecedor porque se pueden establecer espacios de discusión sobre aspectos comunes y de interés compartido. En este, de acuerdo con el autor, el grupo de estudiantes cree que la educación es un proceso de desprisionalización y socialización. Po consiguiente, la comunidad docente facilita nuevas ideas y conocimientos, lo cual contribu- ye a la exploración de nuevas perspectivas con respecto a la vida.

En este orden de ideas, se debe reforzar que la persona docente a distancia y en medios virtuales mantiene su condición de facilitador o de facilitadora de aprendizaje y de conocimientos. La educación a distancia no limita un papel constructivo, participativo ni propositivo del profesorado. Lo esencial es que tenga esto claro y que construya su práctica pedagógica con recursos que faciliten ese papel.

En una enseñanza a distancia los contenidos y los materiales de aprendizaje son elementos esenciales. El otro elemento o factor clave esencial es la interacción tutor-estudiante. Estas características influyen y determinan la eficacia y el éxito de los programas a distancia. La calidad de la comunicación entre el estudiante y el tutor es crucial para el curso de estudios (Saez, Domínguezy Mendoza, 2014, p. 199).

También, se destaca la importancia de la comunidad de funcionarios universitarios en el contexto penitenciario, sea específica o no su función de docencia. El impacto de su trabajo en el contexto de prisión es trascendental y siempre pueden y deben establecerse como facilitadores de conocimiento; aquí el grupo de funcionarios son representantes de nuevas condiciones y posibilidades de vida, del conocimiento y de otros mundos posibles.

En el contexto costarricense y de educación universitaria a distancia en los centros penitenciarios, la persona funcionaria universitaria no docente cumple funciones de entrega y de recepción de información, de acompañamiento en el proceso de aprendizaje y de su evaluación. Asimismo, su actitud y entendimiento crítico de su papel es fundamental para el éxito del modelo pedagógico y del proceso educativo de cada estudiante.

En suma, el modelo pedagógico que se reflexiona aquí debe comprender la importancia 
de la docencia y del acompañamiento, así como promover papeles y responsabilidades coherentes con esta apreciación.

\section{La evaluación de los aprendizajes}

Un apartado básico en la reflexión del modelo pedagógico requiere la evaluación de aprendizajes. En el contexto penitenciario, debe facilitarse y fortalecerse en la medida en que se desarrolle, de manera regulada, autorregulada e integral. Esto involucra que no dependa de acciones puntuales ni ajenas al proceso educativo, sino que deben ser parte constante de este. En este sentido, UNED (2004) destaca que la evaluación de aprendizajes debe "[...] incorporar el concepto de evaluación como regulación y autorregulación de los aprendizajes, de manera que la evaluación llegue a ser integral, durante todo el proceso de aprender, e integrada, es decir, no separada del proceso como momento de comprobación" (p. 17).

En sí, se trata de que el grupo de estudiantes cumpla con los contenidos determinados y pueda dar cuenta de su apropiación y su aprendizaje, sin que la evaluación sea un aspecto desintegrado del proceso educativo. Además, se espera que las personas participantes se apropien de este proceso de evaluación, entendiendo la manera en que se le evalúa y su capacidad de regular su adquisición de conocimiento para su respectiva comprobación.

Dentro de esta perspectiva, la evaluación de los aprendizajes no puede consistir solamente en exigir al estudiante que dé cuenta, en un momento dado, de la información, conceptos o procedimientos que ha sido capaz de retener. En un modelo centrado en el estudiante, resulta esencial diferenciar lo que tradicionalmente se ha denominado "evaluación", identificada con la habilidad de responder pruebas para obtener una calificación, de lo que venimos denominando "regulación de los aprendizajes" (UNED, 2004, p.17).
Para esta forma de evaluación, destaca un profesorado con capacidad para regular el proceso de aprendizaje a partir de habilidades de comunicación, guía y facilitación de la información; el desarrollo del estudiantado en cuanto a la habilidad de regular su proceso de aprendizaje; y la promoción de la coevaluación entre estudiantes para posibilitar la construcción conjunta del aprendizaje (UNED, 2004).

En la cuestión del aprendizaje, se requiere investigación significativa y un modelo que, realmente, promueva, asegure y evalúe, de forma adecuada, el crecimiento académico y profesional ¿Está el estudiantado aprendiendo o solo es partícipe de una oferta educativa?

No está demás decir que el e-learning también suma, y de gran manera, en lo respectivo a la evaluación de aprendizajes. La utilización de las nuevas tecnologías de la información posibilita significativamente que la comunidad de estudiantes sea capaz de regular sus aprendizajes y pueda avanzar en el conocimiento de acuerdo con sus propios procesos de adquisición del saber y con la adecuada y coherente facilitación didáctica y docente.

Para finalizar, en términos generales, la educación en el contexto penitenciario es considerada como una oportunidad de liberación. El modelo pedagógico, la pedagogía en sí, debe de ser liberador; relacionado con Freire y su pedagogía crítica, es una educación que puede significar la oportunidad de crear un ser humano crítico, que problematiza y que, a través de esta problematización, se libera. "La educación problematizadora se hace, así, un esfuerzo permanente a través del cual los hombres van percibiendo, críticamente, cómo están siendo en el mundo, en el que y con el que están" (Freire, 1997b, p.90, citado en Valverde, 2011, p. 126).

En el contexto tan opresivo de la prisión, se tiene que establecer un modelo pedagógico pen- 
sado como liberador. En el discurso manifiesto y falaz de la "adaptación social" y todos los procesos "re" (rehabilitación, resocialización, reintegración), a partir del encarcelamiento, habría que distinguir a la educación y darle un lugar primordial como creador de nuevas posibilidades y de nuevos mundos.

\section{Conclusión}

De acuerdo con el objetivo de explorar elementos trascendentales y necesarios cuando se plantea, se piensa y se reflexiona en un modelo pedagógico de educación a distancia para estudiantes universitarios en prisión, se han presentado y discutido algunas consideraciones fundamentales y que permiten atender, en gran medida, al objetivo de una educación de calidad, la cual atienda a las razones de disponibilidad, accesibilidad, adaptabilidad y aceptabilidad (INEE, 2014).

Aquí destacó el enfoque de derechos humanos; en otras palabras, se debe comprender la educación como un derecho que trasciende la instancia o el contexto en el que se desarrolley, por ende, es prioridad asumir la innegable necesidad de desarrollarlo y de potenciarlo en prisión.

También, la perspectiva de género e inclusividad despuntó. En este sentido, se concluyó la importancia de diseñar procesos educativos y, en específico, modelos pedagógicos que incluyan la diversidad y que potencien las habilidades y las capacidades particulares de todas las personas. Para ello, es requisito alejarse de la reproducción de papeles o estigmas impuestos a algunas poblaciones.

Sobresalió como aspecto imprescindible por considerar en la educación en el contexto carcelario la utilización del e-learning y las nuevas tecnologías de información. El acceso a la información es, por sí mismo, un derecho y, en la academia y el estudio, permite investigar, explorar conocer y comprender. La cotidianidad de un estudiante, más aún universitario, se limita si no puede acceder a contenidos teóricos y ni a materiales didácticos que le permitan profundizar en el aprendizaje.

Por supuesto, un modelo pedagógico para este contexto debe tomar en consideración las necesidades y especificidades de la población en cuestión, de acuerdo con la perspectiva y con el criterio del mismo estudiantado (ver figura 1). El contexto es muy específico y plantea necesidades especiales y diferenciadas de otros espacios. Por lo tanto, no puede reflexionarse acerca de la educación universitaria en este espacio si no se integran dichas especificidades a su proceso. En virtud de lo anterior, se concluye y se asegura que hay muchísimo por hacer en aquellos contextos e instituciones que no hayan atendido, de manera integral, las cuestiones aquí presentadas y el resultado de la investigación.

En Costa Rica específicamente, el estudio en prisión no se ha priorizado como corresponde. Además, está claro que el hecho de que exista la oferta no garantiza el pleno disfrute del derecho a la educación. La oferta de la UNED Costa Rica en este contexto es notablemente valiosa y refleja una voluntad política y un criterio profesional y académico con una perspectiva coherente con los derechos humanos, pero no puede quedarse en esto. La oferta es un primer paso que muy pocas instituciones educativas en el mundo asumen; no obstante, es un primer paso de muchos por dar. Si bien la UNED cuenta con un modelo pedagógico general, este no ha atendido, con rigurosidad y énfasis, las especificidades y necesidades de sus estudiantes privados de libertad y del contexto en donde se desarrollan, lo cual ubica al estudiantado en un escenario de clara desventaja. Por lo tanto, existe la necesidad de estructurar y de diseñar un modelo específico y especializado.

Es inminentemente necesario y se recomienda a las instancias responsables en nuestro país y 


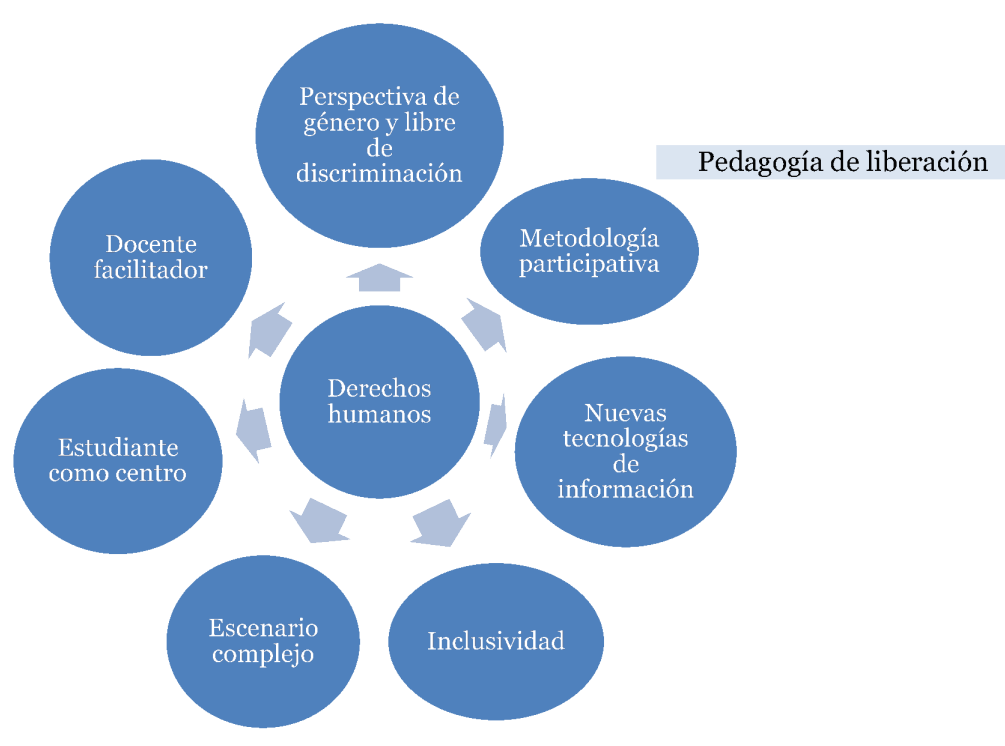

Figura 1. Sistematización de aspectos esenciales de modelo pedagógico. Fuente: elaboración propia.

en aquellos países y universidades con contextos semejantes que se establezcan espacios para la reflexión, la propuesta y la ejecución de mejoras en el sistema educativo universitario en prisión. Esto permite el avance, la innovación, la especialización y, como se ha dicho, sobre todo, garantiza, visibiliza y defiende un derecho.

Con respecto a esto y refiriéndose a la educación en cárceles, di Franco y Domínguez (2005) destacan que

Entender los efectos limitativos podría permitir también encontrar posibilidades de cambio solo con entender las cosas de otra manera, pensar que las situaciones mismas pueden ser cambiadas mediante la transformación de las prácticas que las constituyen, así como de los entendimientos que les dan sentido (p. 160).

Así, este trabajo sirve para replantear los escenarios educativos en el contexto de las prisiones; para dar cuenta de la necesidad de cambios, mejoras e innovación, pero sobre todo, del hecho de que dichos cambios son, no solo necesarios, sino posibles, e incluso reales en algunos lugares del mundo. La tarea es, entonces, comenzar, no solo con la discusión y revisión de lo que se hace, sino con el planteamiento, la estructuración y la puesta en práctica de estrategias y de acciones innovadoras.

Para finalizar, se recomienda la investigación futura dirigida a la construcción y a la especialización de modelos pedagógicos de educación para el contexto de prisión. Se considera, también, importante la investigación y el desarrollo de proyectos profesionales y académicos dedicados a la construcción de materiales y de recursos didácticos para estudiantes en prisión que incorporen las nuevas tecnologías de información.

En resumen, se motiva a las instituciones responsables a abrir espacios de discusión e investigación acerca de cómo se puede incorporar el internet y las destacadas herramientas tecnológicas en los procesos educativos de las prisiones, para potenciar el acceso a la información y, en sí, los procesos académicos que emprende el estudiantado.

\section{Referencias}

Barahona, M. (2012). El papel de la investigación teórica en la construcción del conocimiento: 
una reflexión desde la Universidad Estatal a Distancia (UNED). Revista Rupturas. 3(1), 2-16. Recuperado de http://investiga.uned. ac.cr/revistas/index.php/rupturas/article/ view/254/137

Blazich, G., de Milén, S. y Viedma, A. (2007). La educación en establecimientos penitenciarios argentinos: estudio de algunas cárceles de las ciudades de resistencia y corrientes. Revista digital. 1, 1-25. Recuperado de http:// hum.unne.edu.ar/revistas/educa/archivos/ revista1/archivos/pub 1/blazich rev1.pdf

Cammarosano, E. (2011). O papel da escola na prisão: saberes e experiências de alunos e profesores. Childhood $\mathcal{E}$ Philosophy. 7(14), 271-297. Recuperado de http://www.e-publicacoes.uerj.br/index.php/childhood/article/ view/20571/14897

Caride, J. A. y Gradaille, R. (2013). Educar en las cárceles: nuevos desafíos para la educación social en las instituciones penitenciarias. Revista de educación. 360, 36-47. Recuperado de https://dialnet.unirioja.es/servlet/articulo?co$\underline{\text { digo }=4098029}$

Díaz-Barriga, F. y Hernández, G. (2002). Estrategias docentes para un aprendizaje significativo. Una interpretación constructivista. 2. a edición. México: Mc Graw Hill Interamericana.

Di Franco y Domínguez (2005). Educación secundaria en la cárcel: análisis de una experiencia educativa en la Unidad Penitenciaria N. 4. Revista Anuario N 7. Facultad de Cs. Humanas UNL Pan. 157-175. Recuperado de http:// www.biblioteca.unlpam.edu.ar/pubpdf/anuario fch/n07a13difranco.pdf

Elvira-Valdes, M. y Duran-Aponte, Emilse (2014). Estudiar en mayúsculas: la educación formal en instituciones penitenciarias venezolanas. Revista Sophia [online]. 10(1), 64-73. Recuperado de http://www.scielo. org.co/scielo.php?script=sci arttext\&pi-
$\underline{\mathrm{d}=S 1794-89322014000100006 \& \ln \mathrm{g}=\mathrm{en} \& \mathrm{nrm}=\mathrm{i}-}$ $\underline{\mathrm{SO}}$

Fernández-Morales, F. y Duarte, J. (2016). Retos de la Inclusión Académica de Personas con Discapacidad en una Universidad Pública Colombiana. Formación Universitaria. 9(4), 95104. Recuperado de http://www.scielo.cl/pdf/ formuniv/v9n4/art11.pdf

Hammerschick, W. (2010). Report on E-learning in European Prisons - Concepts, Organisation, Pedagogical Approaches in Prison Education. Learning Infrastructure for Correctional Services. Recuperado de https://ec.europa.eu/epale/ sites/epale/files/report on e-learning in european prisons.pdf

Instituto Nacional para la Evaluación de la Educación (INEE) (2014). El derecho a una educación de calidad. México: INEE. Recuperado de http://publicaciones.inee.edu.mx/buscadorPub/P1/D/239/P1D239.pdf

Miranda, R. (2007). Educación superior e igualdad de género. CPU-e, Revista de Investigación Educativa. 4. Instituto de Investigaciones en Educación Veracruz, México, 1-30. Recuperado de http://www.redalyc.org/articulo. oa?id=283121710004

Moreira, R. y Machado (2017). La educación superior a distancia y el e learning en las prisiones en Portugal. Revista comunicar. 51, 39-49. Recuperado de https://www.revistacomunicar.com/index.php?contenido=detalles\&numero $=51 \&$ articulo $=51-2017-04$

Organización las Naciones Unidas (ONU) (2015). Declaración Universal de los Derechos Humanos. Edición ilustrada. Recuperada de http://www. un.org/es/documents/udhr/UDHR booklet SP web.pdf

Saez, J. M.; Domínguez, C. y Mendoza, V. (2014). Valoración de los obstáculos, ventajas y prácticas del e-learning: un estudio de caso en 
universidades iberoamericanas. Educatio Siglo XXI. 32(2), 195-220. Recuperado de http://revistas.um.es/educatio/article/view/202221

Universidad Estatal a Distancia (UNED) (2004). Modelo Pedagógico de la Universidad Estatal a Distancia. Aprobado por el Consejo Universitario, sesión 1714, artículo IV, inciso 3) del 9 de julio de 2004. San José: EUNED.
Valverde, F. (2011). Pedagogía de la liberación de la educación opresora a la educación liberadora: un vistazo a la educación en el siglo XXI desde Paulo Freire. Pensamiento actual. 11(16-17), 117-131. Recuperado de http://revistas.ucr.ac.cr/index.php/pensamiento-actual/ article/view/10457

Recibido: 04 de agosto de 2017

Aceptado: 19 de octubre de 2017 